\title{
miR-143 is downregulated in cervical cancer and promotes apoptosis and inhibits tumor formation by targeting Bcl-2
}

\author{
LIPENG LIU ${ }^{1}$, XIAOHUA YU ${ }^{2}$, XIAOFANG GUO ${ }^{1}$, ZHI TIAN $^{1}$, MIN SU $^{1}$, YU LONG $^{1}$, CHUNXIA HUANG $^{1}$, \\ FENG ZHOU $^{1}$, MEILING LIU ${ }^{1}$, XINHUA WU ${ }^{3}$ and XIAOCHUN WANG ${ }^{4}$ \\ ${ }^{1}$ Department of Biochemistry and Molecular Biology, Changsha Medical University, Changsha 410219; \\ ${ }^{2}$ Institute of Pharmacy and Pharmacology, Nanhua University, Hengyang, Hunan 421001; \\ ${ }^{3}$ Department of Obstetrics and Gynecology, Xiangya Hospital, Central South University, \\ Changsha 410078; ${ }^{4}$ Department of Medical Laboratorial Examination, \\ Central South University, Changsha 410013, P.R. China
}

Received September 30, 2011; Accepted December 1, 2011

DOI: $10.3892 / \mathrm{mmr} .2011 .696$

\begin{abstract}
RNAs (miRNAs) are small non-coding RNA molecules of 21-24 nt that regulate the expression of other genes by transcriptional inhibition or translational repression. Multiple lines of evidence suggest that miRNAs play important roles in tumor development and progression. We identified 24 miRNAs markedly and aberrantly expressed in human cervical cancer. The most significantly deregulated was miR-143 as determined by miRNA microarray analysis. miR-143 was introduced into HeLa cells and it was found that the overexpression of miR-143 significantly inhibited HeLa cell proliferation and promoted apoptosis; anti-miR-143 rescued the effects. HeLa cells transfected with pre-miR-143, pre-anti-miR-143 or control miRNA precursor were injected subcutaneously into the flanks of female athymic nude mice, and the overexpression of miR-143 suppressed the formation of tumors. Compared with normal cervical tissues, the levels of $\mathrm{Bcl}-2$ were increased in miR-143-downregulated tissues. Sustained overexpression of miR-143 in HeLa cells resulted in suppression of $\mathrm{Bcl}-2$ expression, and knockdown of miR143 by anti-miR-143 increased Bcl-2 expression. In addition, overexpression of $\mathrm{Bcl}-2$ partially reversed the inhibition of proliferation and promotion of apoptosis in the HeLa cells caused by miR-143. Furthermore, miR-143 suppressed the activity of a luciferase reporter carrying the 3'-UTR of Bcl-2, which was abolished by mutation of the predicted miR-143binding site, indicating that $\mathrm{Bcl}-2$ is a miR-143 target gene. Our study revealed a molecular link between miR-143 and $\mathrm{Bcl}-2$. Direct involvement in the regulation of $\mathrm{Bcl}-2$ may be
\end{abstract}

Correspondence to: Dr Xiaochun Wang, Department of Medical Laboratorial Examination, Central South University, Changsha 410013, P.R. China

E-mail:wangxiaochun65@yeah.net

Key words: miR-143, Bcl-2, HeLa, proliferation, apoptosis one of the mechanisms through which miR-143 may play a role in the pathogenesis of cervical cancer.

\section{Introduction}

microRNAs (miRNAs) are endogenous, small, single-stranded, non-coding RNAs that regulate the expression of a wide variety of genes by transcriptional inhibition or translational repression. Therefore, miRNAs function in diverse physiological and pathological processes. Since altered expression of miRNAs is reported in various types of cancers, it is reasonable to hypothesize that miRNAs play important roles in tumor development and progression. In fact, numerous studies have shown that miRNAs are involved in cell proliferation, apoptosis (1), differentiation (2), reproduction (3) and cancer (4). In addition, miRNAs also function as potential oncogenes or tumor-suppressor genes involved in tumor development and progression, and this has been well documented in many tumors, including pancreatic (5), colon (6), gastric (7), breast (8), liver (9), lung (10) and nasopharyngeal cancer (11).

Cervical cancer is the second most common malignancy in women worldwide, resulting in approximately 300,000 deaths each year. Yet, the underlying mechanisms of cervical cancer pathogenesis are still ill-defined. Emerging evidence indicates that miRNAs play a virtual role in the development of different types of cancers including cervical cancer. Wang et al (12) identified several aberrantly expressed miRNAs in cervical cancer. The expression of miR-143 was not only downregulated in cervical cancer tissue but also was lower in cervical cancer cell lines. Of particular interest, overexpression of miR-143 in HeLa cells successfully suppressed cell growth. Martinez et al (13) also suggested that the effect of miR-143 on cervical carcinogenesis is HPV-independent.

We are particularly interested in miR-143 and cervical cancer development. Using the programs RNA22, PICTAR4, PICTAR5, PITA, TargetScan, miR-143 was predicted to be able to interact with the 3'-UTR of Bcl-2 mRNA. It has been reported that $\mathrm{Bcl}-2$ is sensitive to miR-143 in HCT116 human colorectal cancer cells (14), and mechanistically miR-143 
targeted Bcl-2 in its 3'-UTR region. Firstly, we showed that miR-143 was downregulated in cervical cancer tissues. Secondly, overexpression of miR-143 markedly inhibited HeLa cell proliferation in vitro and in vivo. Overexpression of Bcl-2 partially reversed the inhibition of HeLa cell proliferation caused by miR-143. These findings suggest that miR-143 may play a role in the pathogenesis of cervical cancer through the regulation of the expression of $\mathrm{Bcl}-2$.

\section{Materials and methods}

Samples. Pair-matched tumor and adjacent non-tumor cervical tissues from 15 patients were obtained from Xiangya Hospital, Central South University (Changsha, China) at the time of surgery during the period between May and October, 2009. Ethical approval for use of all specimens was obtained from the Research Ethics Committee of the Xiangya Hospital. Written informed consent was obtained before collection. All of the patients were diagnosed with primary cervical cancer according to formal pathological reports. The samples were snap frozen immediately and stored in liquid nitrogen for RNA and protein extraction. All specimens used in the analyses consisted of $>90 \%$ tumor cells, as examined by a gynecologic pathologist.

Cell culture and tissue miRNA microarray analysis. HeLa cells (Shanghai Bioleaf Biotechnology Company, China) were cultured in RPMI-1640 medium (Invitrogen, USA) supplemented with $10 \%$ fetal bovine serum (FBS, Hyclone, USA), $2.05 \mathrm{mmol} / \mathrm{l} \mathrm{L}$-glutamine, $100 \mathrm{U} / \mathrm{ml}$ of penicillin and $100 \mu \mathrm{g} / \mathrm{ml}$ streptomycin at $37^{\circ} \mathrm{C}$, in $5 \% \mathrm{CO}_{2}$. Total-RNA was extracted from the cancer tissues using TRIzol (Invitrogen) according to the manufacturer's instructions, and miRNAs were further purified using the MirVana miRNA Isolation kit (Ambion, Austin, TX, USA). The miRNA expression profiles were analyzed using MirVana miRNA Bioarray. Each array contains probes for all miRNAs in quadruplicate, and the signals obtained for each miRNA are represented as an average of four experiments. The small RNA fractions were fluorescently labeled and hybridized to the Bioarrays according to the manufacturer's instructions (Ambion). Each experiment was conducted twice. The Bioarrays were scanned using GenePix 4000B Scanner, and the median fluorescent intensity was obtained after subtracting the background using GenePix Pro 6.0 software.

Quantitative real-time PCR and RT-PCR. The expression of the miRNAs was estimated using TaqMan MicroRNA assays (Applied Biosystems) with specific primers for miR-143 (P/N: 4373134, Applied Biosystems) in both the tissue samples and cultured cells. cDNA was reverse-transcribed from $10 \mathrm{ng}$ of total RNA samples using the looped primers from the TaqMan MicroRNA assays and reagents from the TaqMan MicroRNA Reverse Transcription kit (Applied Biosystems). Real-time PCR was performed using the standard TaqMan miRNA assay protocol on an ABI7300 real-time PCR detection system. The snRNA U6 was used to normalize the expression levels of each miRNA. Bcl-2 mRNA was detected by RT-PCR. Total-RNA was isolated from cells using TRIzol (Invitrogen) and used for the firststrand cDNA synthesis with Superscript ${ }^{\mathrm{TM}}$ Reverse transcriptase and oligo(dt)18 primer according to the manufacturer's protocol (Life Technologies, Gaithersburg, MD, USA). Bcl-2 sense primer was 5'-CGACGACTTCTCCCGCCGCTACCGC-3' and antisense primer, 5'-CCGCTAGCTGGGGCCGTACA GTTCC-3'. The expected size of the product is $318 \mathrm{bp}$. $\beta$-actin was used as the internal control. $\beta$-actin sense primer was 5'-CATCCTGCGTCTGGACCT-3' and antisense primer, 5'-CAGGAGGAGCAATGATCTTG-3' with a predicted product of $480 \mathrm{bp}$. The PCR products were separated on $1.5 \%$ agarose gel.

Construction of the plasmids and transfection. To construct the plasmid expressing miR-143, synthesized precursor miR-143 (pre-miR-143) and antisense-miR-143 (pre-antimiR-143) (Cell Biolabs, USA) were inserted into the miRNASelect ${ }^{\mathrm{TM}}$ pEGP-miR vector (Cell Biolabs). The control miRNA was inserted similarly into the miRNASelect ${ }^{\mathrm{TM}} \mathrm{pEP}-\mathrm{miR}$ vector (Cell Biolabs). All the vectors were capable of expressing the GFP biomarker. Positive clones were selected using puromycin, and finally, qRT-PCR was performed to verify indirectly the sequence of miR-143. For overexpression of $\mathrm{Bcl}-2$, the open reading frame of the $\mathrm{Bcl}-2$ was inserted into the pCMV6-AC-RFP vector (OriGene, USA). To construct the luciferase reporter, the 3'-UTR of the Bcl-2, containing the miR-143-binding sequence (CAUCUCA, 1811-1817 bp) was amplified by RT-PCR; sense primer, 5'-CCGTGTTTAATGCTGTGGTTGA-3' and antisense primer, 5'-GCCAGTGACATCCAGGTTTTTCTTA-3' with a predicted product of $229 \mathrm{bp}$, was inserted into the pMIRREPORT $^{\mathrm{TM}}$ miRNA Expression Reporter Vector from Ambion (pMIR-Bcl-2). The pMIR-Bcl-2-mut was constructed in a similar way except the A's in the CAUCUCA were mutated into G's using a Site-Directed Mutagenesis kit (Stratagene, USA) according to the manufacturer's instructions. The HeLa cells were seeded in 6 -well plates at $20 \times 10^{4}$ cells/well and after growing overnight to $70-80 \%$ confluence, the cells were transfected with the different plasmids.

MTT assay. The cells were suspended in $200 \mu 1$ medium and seeded in 96 -well plates $\left(1 \times 10^{4} / \mathrm{ml}\right)$ and grown to $50 \%$ confluence and synchronized with serum-free medium for $24 \mathrm{~h}$ before the serum concentration was brought to $10 \%$. Then $20 \mu \mathrm{l}$ MTT reagent $(5 \mathrm{mg} / \mathrm{ml}$; Amresco, USA) was added at different time points $(24,48$ and $72 \mathrm{~h})$. After incubation at $37^{\circ} \mathrm{C}$ for $4 \mathrm{~h}$ and a brief centrifugation, $150 \mu \mathrm{l}$ of DMSO was added to halt the reaction. The cell confluence was determined by the absorbance at $570 \mathrm{~nm}$.

Apoptosis assay. Cell death as a result of apoptosis was evaluated using a cell death detection ELISA-Plus kit (Roche Molecular Biochemicals, USA). HeLa cells infected with pre-miR-143, pre-anti-miR-143 or control miRNA precursor were plated in triplicate in 6-well plates. Cells were serum-deprived for $24 \mathrm{~h}$ and then lysed, and the cytoplasmic histone-associated DNA fragmentation (mono- and oligonucleosomes) was detected by spectrophotometry according to the manufacturer's instructions.

Xenograft tumor model. To evaluate in vivo tumorigenesis, a cervical cancer xenografting mouse model was used. 
Table I. Differentially expressed miRNAs in cancerous tissues.

\begin{tabular}{lcc}
\hline microRNA & Fold-change & P-value \\
\hline Overexpressed & & \\
miR-21 & 7.2 & 0.0327 \\
miR-17-5p & 6.5 & 0.0038 \\
miR-205 & 6.4 & 0.0029 \\
miR-199a & 6.2 & 0.0231 \\
miR-16 & 5.8 & 0.0329 \\
miR-199b & 5.5 & 0.0045 \\
let-7f & 5.1 & 0.0037 \\
miR-214 & 4.7 & 0.0403 \\
miR-210 & 4.5 & 0.0289 \\
miR-9 & 4.0 & 0.0021 \\
miR-27a & 3.7 & 0.0032 \\
miR-31 & 3.5 & 0.0397 \\
miR-193b & 3.2 & 0.0025 \\
miR-193c & 3.1 & 0.0078 \\
miR-34a & 2.6 & 0.0367 \\
miR-200c & 2.5 & 0.0253 \\
miR-127 & 1.8 & 0.0036 \\
miR-133a & 1.5 & 0.0027 \\
Underexpressed & & 0.0089 \\
miR-143 & -8.4 & 0.0421 \\
let-7c & -7.1 & 0.0236 \\
miR-203 & -5.3 & \\
miR-196b & -3.7 & \\
miR-149 & & \\
miR-145 & & \\
\hline & & \\
\hline
\end{tabular}

Female athymic nude mice (nu/nu, 3 to 4-weeks old) were purchased from Shanghai Laboratory Animal Co., Ltd. (SLAC, Shanghai, China) and maintained in the Laboratory for Experiments, Central South University under laminar airflow conditions. The studies were conducted in accordance with the standards established by the Guidelines for the Care and Use of Laboratory Animals by Central South University. All procedures involving mice were approved by the Central South University Animal Management Committee. HeLa cells $\left(5 \times 10^{6}\right)$ transfected with pre-miR-143, pre-anti-miR-143 or control miRNA precursor were injected subcutaneously into the flanks of nude mice (6 mice/group). The volume (V) of the implanted tumor was measured every 3rd day with a vernier caliper, and the tumor sizes were estimated using the formula: $\mathrm{V}\left(\mathrm{mm}^{3}\right)=$ length $\mathrm{x}$ width ${ }^{2} \mathrm{x} 0.5$. Tumor xenografts were harvested, weighed and snap-frozen 4 weeks after inoculation.

Western blot analysis. The protein lysate was separated by $8 \%$ SDS-PAGE and transferred onto a PVDF transfer membrane (Bio-Rad, USA). After blocking in a $10 \%$ skim milk solution, membranes were incubated overnight at $4^{\circ} \mathrm{C}$ with anti-Bcl-2 (Santa Cruz Biotechnology, USA). Then they were further incubated for $45 \mathrm{~min}$ with goat anti-rabbit horseradish peroxidase (HRP)-coupled secondary antibodies (Boster, China) at room temperature. Immunoreactive proteins were detected by

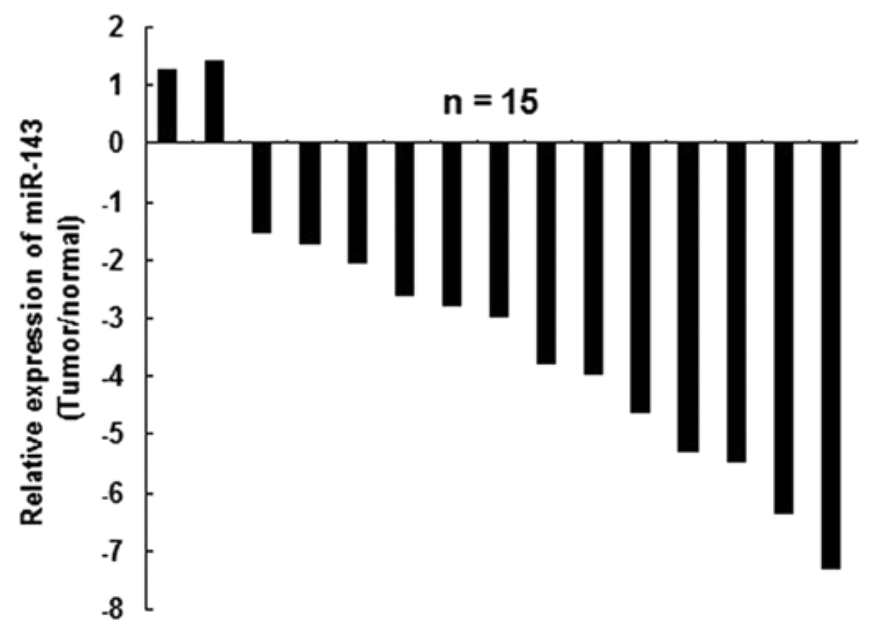

Figure 1. miR-143 is commonly downregulated in cancerous cervical tissues. qRT-PCR was used to estimate the relative expression of miR-143 in 15 primary cervical cancer tissues using the TaqMan miRNA assays with specific primers for miR-143 and snRNA U6. snRNA U6 was used to normalize the expression levels of miRNA. Data are shown as $-\Delta \Delta$ Ct values.

the enhanced chemiluminescence reagent (Amersham, USA), and the chemilluminescent signal on the blot was exposed to X-Omat BTFilm (Kodak, China). The density of the band was quantified and $\beta$-actin was used as the internal standard for all membranes.

Luciferase activity assay. HeLa cells were seeded in 24-well plates $24 \mathrm{~h}$ prior to transfection. pMIR-Bcl-2 or pMIR-Bcl-2-mut luciferase vectors were co-transfected with a control pRL-TK vector (Promega, Madison, WI, USA) into HeLa cells together with pre-miR-143 or control miRNA. The pRL-TK vector (Promega) containing Renilla luciferase was an internal reference. Firefly and Renilla luciferase activities were measured using the dual-luciferase reporter assay (Promega) $24 \mathrm{~h}$ after transfection. Firefly luciferase activity was normalized to Renilla luciferase activity. The experiments were performed in triplicate.

Statistical analyses. Differential expression of miRNA in the surgical samples was determined by testing the null hypothesis that the mean of the $\log ^{2}$-transformed values of the fold change was equal to 0 by one-sample t-test. The data were analyzed by one-way analysis of variance and the Student's t-test to determine statistical significance using SPSS 16.0 statistic software. Each experiment was repeated at least three times. The results were expressed as mean \pm SD. Outcomes were considered statistically significant with two-tailed $\mathrm{P}<0.05$.

\section{Results}

miR-143 is downregulated in cancerous cervical tissues. To investigate the miRNA expression profile of cervical cancer, we initially collected 15 pairs of primary cancerous cervical tissues and their pair-matched non-tumor tissues, and compared their miRNA expression by miRNA microarray analysis. A total of 24 miRNAs showed significantly differential expression in cancerous cervical tissues when compared to the matched 
A

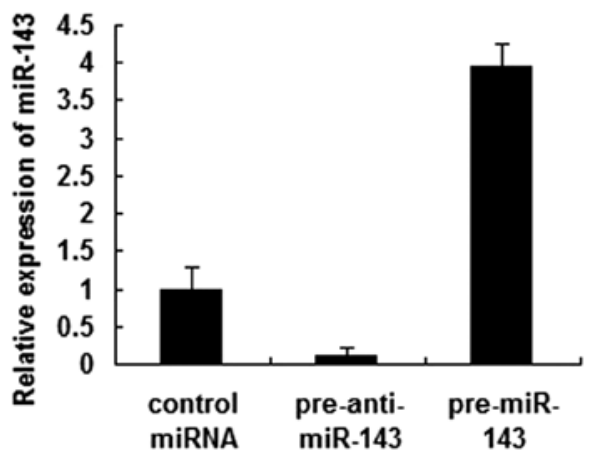

B

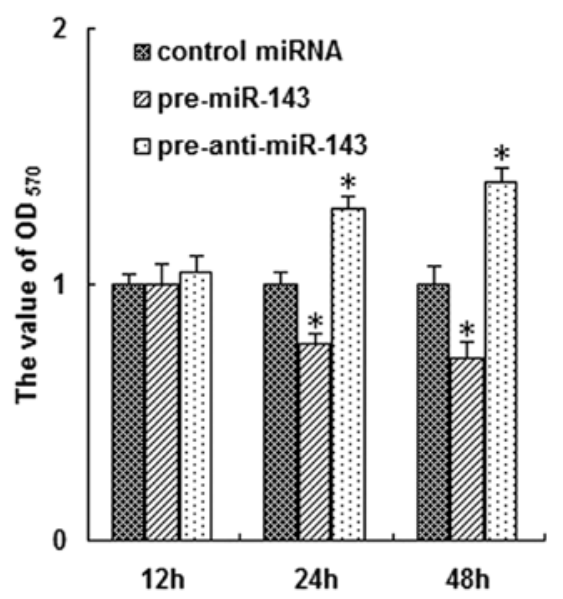

C

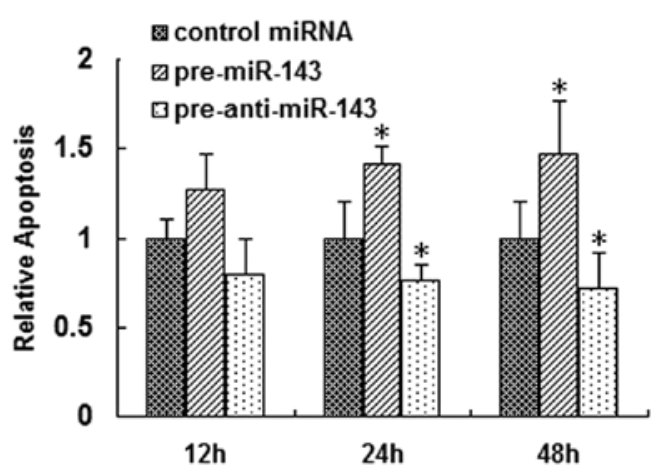

Figure 2. miR-143 affects HeLa cells in vitro. (A) miR-143 expression was measured by qRT-PCR in the selected stable cell lines after transfection with pre-miR-143, pre-anti-miR-143 or control miRNA precursor. snRNA U6 levels were used to normalize the loading difference. (B) HeLa cells transfected with the above plasmids and selected stable cell lines were subjected to MTT analysis at different time periods (12, 24 and $48 \mathrm{~h})$. The cell confluence was determined by the absorbance at $570 \mathrm{~nm}$. (C) Histone-associated DNA fragmentation was assessed by cell death enzyme-linked immunosorbent assay in HeLa cells with transient transfection of pre-miR-143, pre-anti-miR-143 or control miRNA precursor. The relative apoptosis was detected by a microplate reader at $405 \mathrm{~nm}$. Data are shown as mean \pm SD from three independent experiments. ${ }^{*} \mathrm{P}<0.05$ compared with the control miRNA.

non-tumor tissues. miR-16, miR-21, miR-205 and miR-199a were overexpressed and miR-143, let-7c, miR-203, miR-196b, miR-149 and miR-145 were downregulated (Table I). miR-143 was also found to be the most downregulated in the cancerous cervical tissues. To further confirm our findings, the levels of
A

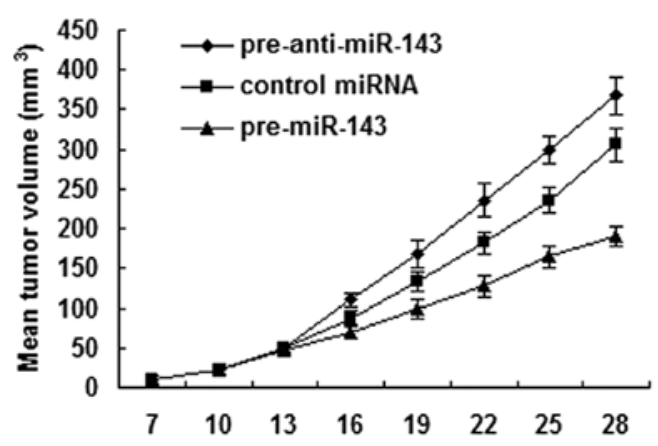

B

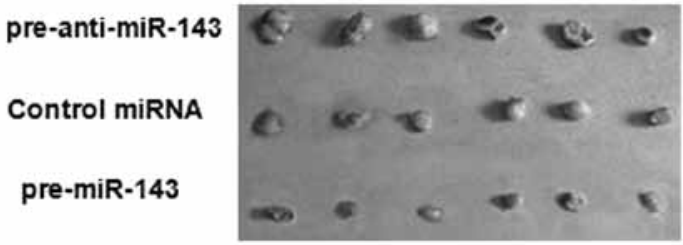

C

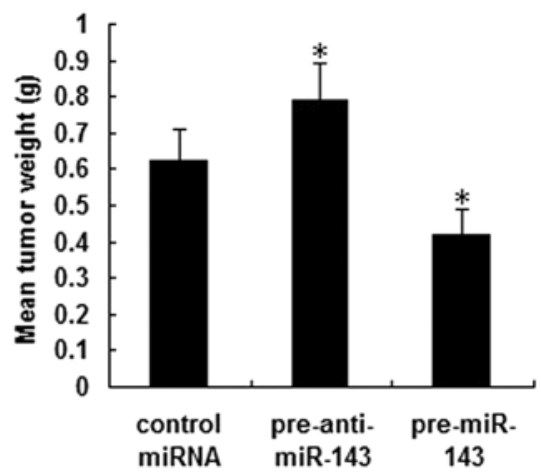

Figure 3. miR-143 suppresses xenograft tumor formation. HeLa cells were transfected with pre-miR-143, pre-anti-miR-143 or control miRNA precursor, and stable cell lines were selected by puromycin. The three cell lines were injected subcutaneously into the flanks of nude mice $(n=6)$. (A) The volume of the tumors was measured every 3 days. The data are shown as mean \pm SE. (B) The mice were sacrificed at 4 weeks after inoculation. Images of the tumors which developed in each group are shown. (C) Weight of the tumors. The data are indicated as mean $\pm \mathrm{SE}$. ${ }^{*} \mathrm{P}<0.05$ compared with the control miRNA.

miR-143 in the cancer tissues of 15 patients were validated by qRT-PCR. We found that miR-143 was downregulated in $86.7 \%$ of these cases ( 13 of 15 patients, $\mathrm{P}<0.01)$ (Fig. 1). These data suggest that miR-143 is frequently downregulated in cancerous cervical tissues.

miR-143 inhibits HeLa cell proliferation and promotes apoptosis in vitro. HeLa cells were transfected with plasmids expressing pre-miR-143, pre-anti-miR-143 or control miRNA precursor, respectively. qRT-PCR analysis validated that the plasmids had been integrated into the genome of the HeLa cells. More importantly, expression of miR-143 was significantly increased in the HeLa cells transfected with pre-miR-143 and was decreased in cells transfected with pre-anti-miR-143 compared to the control miRNA precursor (Fig. 2A).

To evaluate the effects of miR-143 on HeLa cells, we explored the changes in cell proliferation and apoptosis (Fig. 2B and C). Cell proliferation of the miR-143-transfected 
$\mathbf{A}$
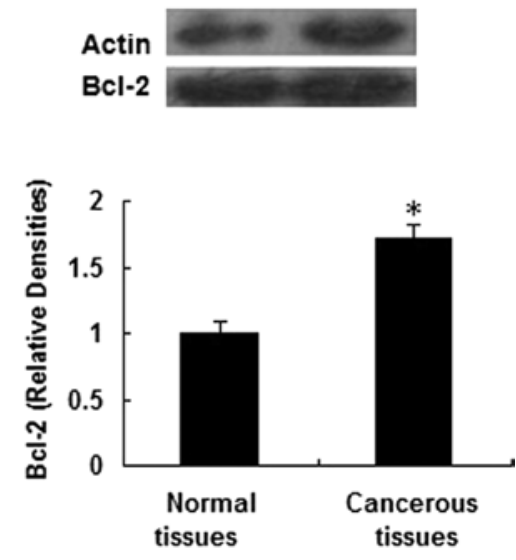

C
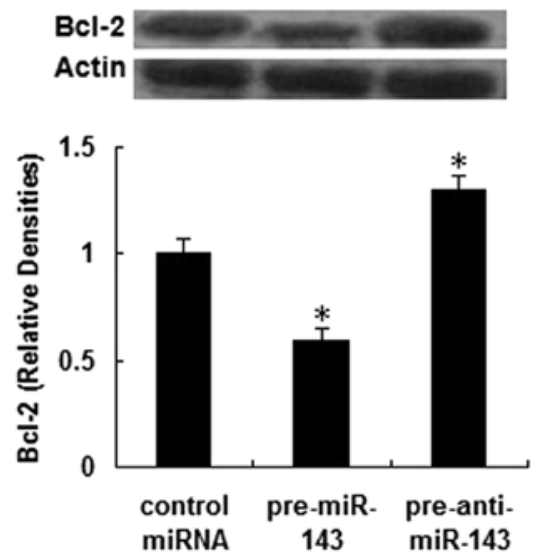

B
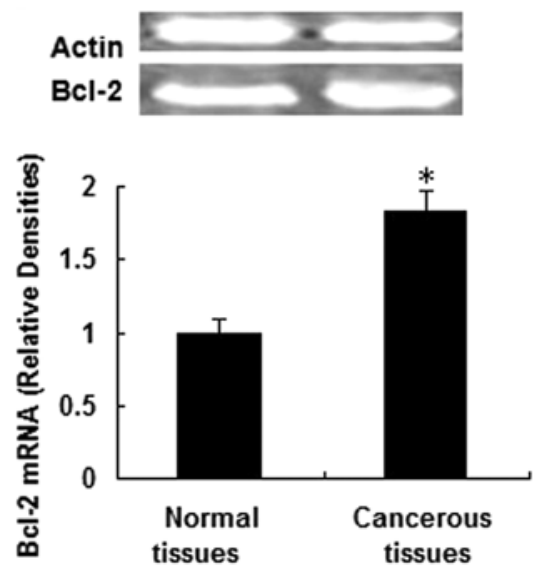

D
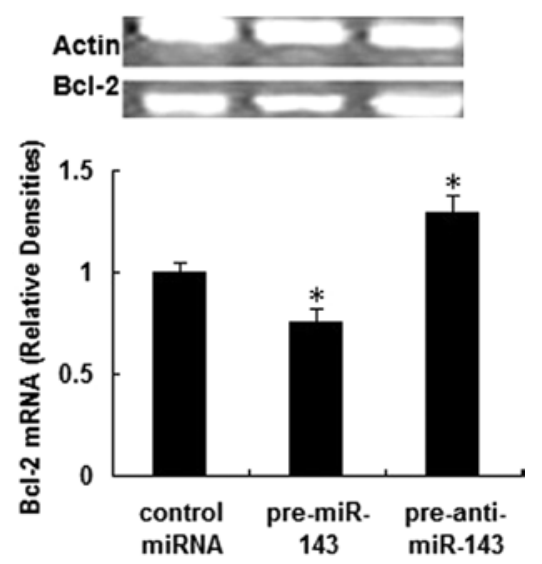

Figure 4. miR-143 inhibits Bcl-2 expression. (A) The expression of Bcl-2 protein in miR-143-downregulated cancerous tissues was measured by western blot analysis. (B) The levels of Bcl-2 mRNA were determined in cancerous tissues by RT-PCR. (C and D) HeLa cells were transfected with pre-miR-143, pre-antimiR-143 or control miRNA precursor, and stable cell lines were selected using puromycin. (C) The expression of Bcl-2 protein in the three cell lines was analyzed by western blot analysis. (D) The levels of Bcl-2 mRNA were subjected to RT-PCR analysis. Each experiment was repeated at least three times, and each sample was assayed in triplicate. ${ }^{*} \mathrm{P}<0.05$ compared with the control miRNA.

HeLa cells was decreased by $13.54 \%$ at $24 \mathrm{~h}, 18.63 \%$ at $48 \mathrm{~h}$, and the relative apoptosis was increased by $75.34 \%$ and $80.81 \%$, respectively; while cell proliferation of the anti-miR143-transfected group was increased by $9.38 \%$ at $24 \mathrm{~h}$ and $13.66 \%$ at $48 \mathrm{~h}$, and the relative apoptosis was decreased by $23.97 \%$ and $27.90 \%$, respectively. The results revealed that miR-143 significantly inhibited the proliferation of HeLa cells and promoted apoptosis, whereas anti-miR-143 had opposite effects. Compared to the control, miR-143 modestly affected the proliferation and apoptosis at $12 \mathrm{~h}$, but had a strong affect at $48 \mathrm{~h}$. This suppressive or enhancing effect was not an immediate cell response. These results indicate that miR-143 may play a role in the proliferation and apoptosis of HeLa cells in vitro; anti-miR-143 partially reverse the role, and this effect required an interval of time.

miR-143 suppresses xenograft tumor formation. Since miR-143 inhibited HeLa cell proliferation in vitro, we further assessed its effect on tumor formation in vivo. HeLa cells $\left(5 \times 10^{6}\right)$ were first transfected with pre-miR-143, pre-anti-miR-143 or control miRNA precursor, respectively, and the stable cell lines were selected by puromycin. The three cell lines were injected subcutaneously into the flanks of nude mice. The volume of the implanted tumor was measured every 3 days. Tumor xenografts were collected and weighed 4 weeks after inoculation. miR-143 markedly reduced tumor size (Fig. 3A and B) and tumor weight (Fig. 3C). However, anti-miR-143 partially reversed the effect. Our data suggest that miR-143 suppresses xenograft tumor formation in HeLa cells in vivo.

miR-143 inhibits Bcl-2 expression. In order to investigate the molecular mechanism of miR-143 action in cervical cancer, the prediction programs RNA22, PICTAR4, PICTAR5, PITA and TargetScan were used, and miR-143 was predicted to be associated with Bcl-2 mRNA at its 3'-UTR. Bcl-2 was further studied as a potential target. We examined the expression levels of Bcl-2 protein and mRNA in miR-143 downregulated tissues, compared to the normal cervical tissues. The expression levels of Bcl-2 protein and mRNA were increased in all tissues with miR-143 downregulation (Fig. 4A and B). In order to further confirm the effect of miR-143 on Bcl-2, we detected the expression of Bcl-2 in HeLa cells transfected with pre-miR-143, pre-anti-miR-143 or control miRNA precursor, respectively. As shown in Fig. 4C and D, compared with the control, the Bcl-2 protein and mRNA levels were significantly reduced in the miR-143-transfected HeLa cells. On the other hand, the levels of Bcl-2 were clearly increased in the HeLa cells transfected with anti-miR-143. Our results suggest that the expression of miR-143 and Bcl-2 have an opposite relation, and miR-143 may inhibit the expression of Bcl-2. 
A

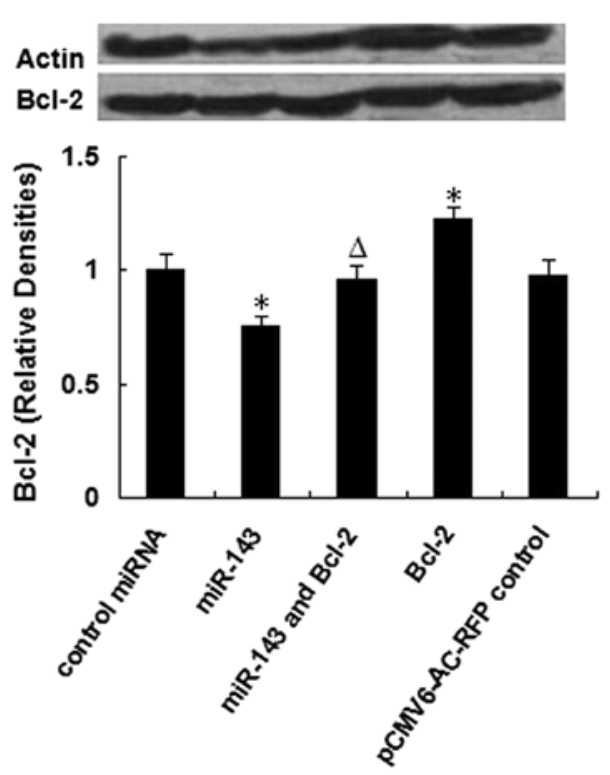

C

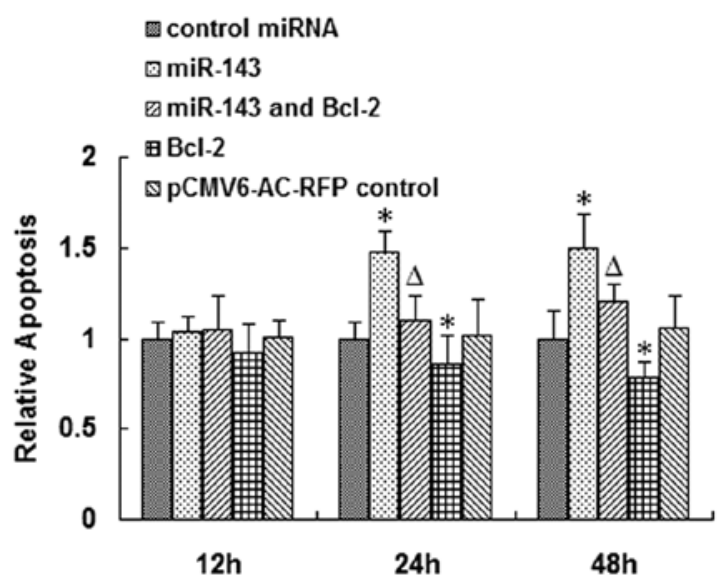

B

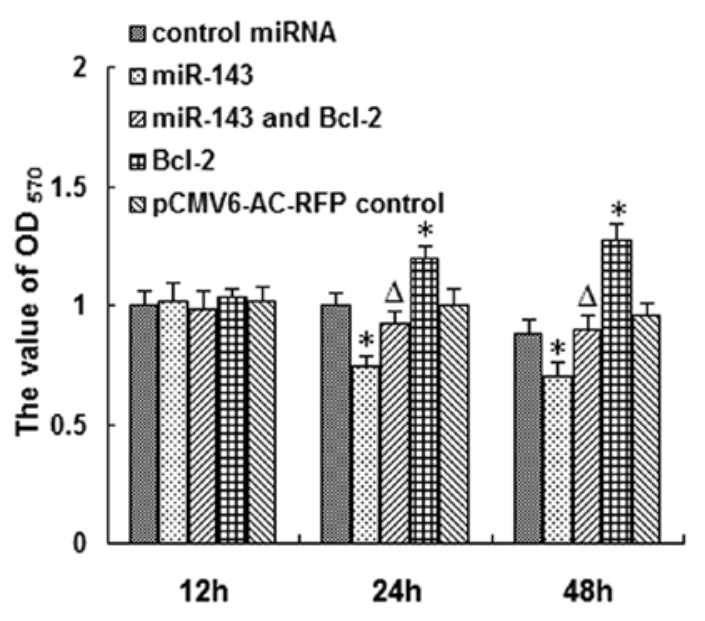

D

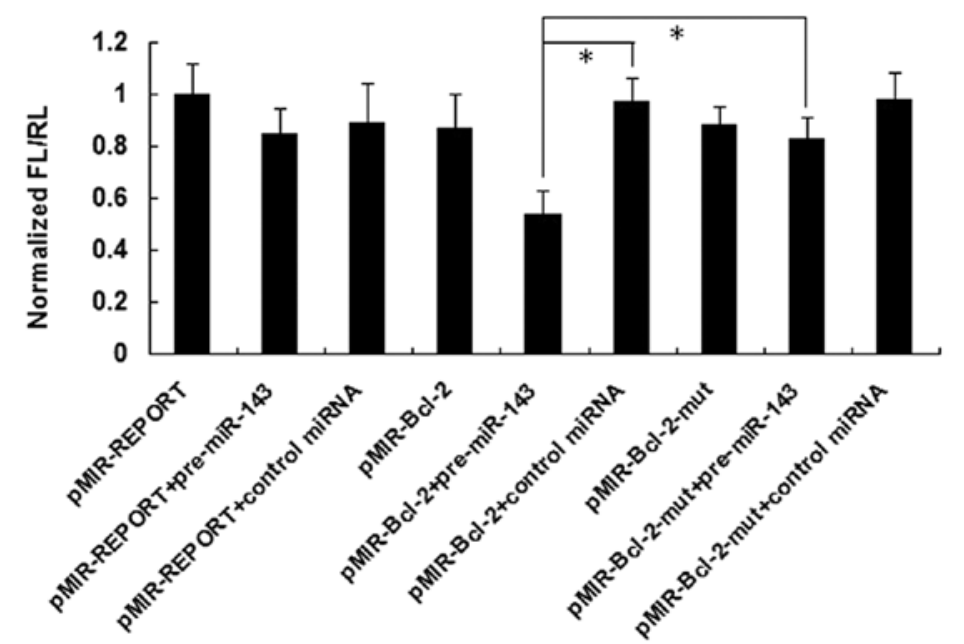

Figure 5. Bcl-2 is a target of miR-143. (A-C) HeLa cells and miR-143-transfected HeLa cells were transfected by the Bcl-2 overexpresssion plasmid, respectively. $\mathrm{P}<0.05$ compared with the control; ${ }^{\Delta} \mathrm{P}<0.05$ compared with Bcl-2-transfected HeLa cells. (A) The levels of Bcl- 2 protein were increased in the HeLa cells with the Bcl-2 overexpression plasmid, whereas the effect of this protein was attenuated by miR-143. The level of actin was used as a loading control. (B) MTT assays showed the changes in proliferation. Bcl-2 promoted cell proliferation, while miR-143 reduced the effect. (C) Histone-associated DNA fragmentation was assessed by cell death enzyme-linked immunosorbent assay in the above cells, which had the opposite effect with the MTT assays. (D) HeLa cells were transfected with the pRL-TK-containing Renilla luciferase gene and indicated vectors or precursors. Bars indicate the Firefly luciferase activities normalized to Renilla luciferase activities of the co-transfected pRL-TK vector. Each experiment was repeated at least three times, and each sample was assayed in triplicate.

$B c l-2$ is a target of miR-143. To test whether miR-143 regulates Bcl-2 directly, we expressed Bcl-2 alone or together with miR-143 in HeLa cells. Western blotting further verified that the Bcl-2 protein was highly expressed in the Bcl-2-transfected HeLa cells (Fig. 5A). More importantly, miR-143 attenuated the Bcl-2 levels. Furthermore, results from the MTT assay showed the effect, of Bcl-2 on cell proliferation (Fig. 5B). The proliferation of the miR-143-transfected HeLa cells was reduced, while the proliferation of the $\mathrm{Bcl}$-2-transfected $\mathrm{HeLa}$ cells was significantly increased by $11.40 \%$ at $24 \mathrm{~h}$ and $20.43 \%$ at $48 \mathrm{~h}$, and the effect mediated by Bcl-2 was weakened when the HeLa cells were co-transfected with pre-miR-143. There were no obvious differences between the control miRNA and pCMV6-AC-RFP controls. The apoptotic results had an oppo- site trend to the Bcl-2 levels (Fig. 5C); apoptosis was inhibited by $13.89 \%$ at $24 \mathrm{~h}$ and $21.21 \%$ at $48 \mathrm{~h}$ when Bcl-2 was overexpressed. However, when the HeLa cells were co-transfected with pre-miR-143, the Bcl-2-mediated effects were clearly weakened. Together with the results from Fig. 4, these data suggest that Bcl-2 may be a target of miR-143.

In order to validate the predicted miR-143-binding sites in the 3'-UTR of the Bcl-2 mRNA, the Bcl-2 luciferase reporter assays were employed. The pMIR-Bcl-2 luciferase vector was transfected into the HeLa cells together with either the control miRNA or the pre-miR-143. Compared with the control miRNA, transfection of the pre-miR-143 significantly decreased the reporter luciferase activity (Fig. 5D). In addition, mutation of the seed region abrogated the suppression of 
luciferase activity caused by miR-143 (Fig. 5D). In contrast, the luciferase activity in cells transfected with pMIR-REPORT firefly luciferase reporter control vector without the 3'-UTR of the Bcl-2 mRNA was not affected. These data strongly suggest that Bcl-2 is a target of miR-143.

\section{Discussion}

It has been reported that miR-143 is downexpressed in colon (15), lung (16) and prostate cancer (17) and is overexpressed in hepatocarcinoma (18). Furthermore, miR-143 is involved in the regulation of MYO6 expression in prostate cancer (17); extracellular signal-regulated kinase-5 (ERK-5) is a miR-143 target in prostate cancer and miR-143 inhibits the proliferation at least in part by the inhibition of ERK-5 activity (19). Restoration of miR-143 expression in colon cancer cell lines was forced to not only decreased tumor cell growth and soft-agar colony formation, but also downregulated the DNMT3A at both the mRNA and protein levels (20). We were interested in understanding the effects of miR-143 on cervical cancer. Firstly, we demonstrated that, compared with the surrounding non-tumor tissues, miR-143 was underexpressed in cancerous cervical tissues. Secondly, miR-143 was demonstrated to inhibit HeLa cell proliferation and promote apoptosis. Finally, we demonstrated that overexpression of miR-143 suppressed tumor formation in vivo. Overexpression of miR-143 lowered Bcl-2 levels. The anti-miR-143 attenuated the effect induced by miR-143. Through the use of reporters, the miR-143 binding site on the 3'-UTR of Bcl-2 appeared to be the predominant site. Finally, we showed that miR-143 inhibited HeLa cell proliferation and promoted apoptosis partially by targeting Bcl-2.

Recent studies have identified that miRNAs play important roles in cervical cancer. Cheng et al (21) found that miR-218 inhibited the proliferation of HeLa cells and induced apoptosis. In their study, miR-214 was downregulated in cervical cancer compared with normal tissue. Overexpression of miR-214 in HeLa cells also significantly inhibited cell proliferation. In addition, HeLa cells that stably overexpressed miR-214 downregulated the expression of MEK3 and JNK1 at both the mRNA and protein levels by targeting the 3'UTRs of these genes (22). Finally, it has been reported that miR-34a inhibits invasiveness through regulation of the Notch pathway and its downstream matrix degrading enzyme in cervical cancer (23). In our study, we found that miR-143 was underexpressed in $86.7 \%$ of the cancerous cervical tissues compared with their non-tumor counterparts. This is consistent with other reports $(12,13)$. Furthermore, miR-143 not only suppressed HeLa cell proliferation and promoted apoptosis, but also inhibited the tumor formation in nude mice. Our data clearly showed that overexpression of miR-143 significantly decreased Bcl-2 expression, and anti-miR-143 elevated it, which indicate that miR-143 regulates expression of Bcl-2. Given the widely reported effects of miR-143 in different tumors, it may be developed as a novel tumor biomarker for diagnosis. Zhang et al (24) found that miR-143 was downregulated in osteosarcoma cell lines and primary tumor samples, and the restoration of miR-143 reduced cell viability, promoted cell apoptosis and suppressed tumorigenicity. Additionally, Bcl-2 was identified to be a direct target of miR-143. Taken together, since the expression of miR-143 and Bcl-2 is inversely related in different types of cancers, these two biomarkers could be used simultaneously to increase diagnostic accuracy.

It is well established that Bcl-2 is an apoptosis-related protein. Overexpression of $\mathrm{Bcl}-2$ is closely related to multiple epithelial tumors resulting from DNA damage and continued survival and subsequently aggregated mutations. Furthermore, the presence of the $\mathrm{Bcl}-2$ protein is strongly associated with the development of invasive cervical disease (25) and Bcl-2 was significantly correlated to the grades of cervical intraepithelial neoplasia (26). The apoptotic effect of miR-29 is Mcl-1- and Bcl-2-dependent (27). It has been suggested that miR-181a, a regulator of $\mathrm{Bcl}-2$, may be targeted to enhance the effect of radiation treatment for malignant glioma cells (28). Both miR-15b and miR-16 could play an important role in the development of MDR in gastric cancer cell apoptosis via Bcl-2 (29). Here, we confirmed that the overexpression of Bcl-2 is associated with cervical cancer. Furthermore, overexpression of Bcl-2 showed similar effects as anti-miR-143 expression, which was attenuated by the overexpression of miR-143. Bcl-2 was confirmed to be a target of miR-143 in this study. This indicates that the suppressive effects of miR-143 in HeLa cell proliferation and promotion of apoptosis is, at least in part, through suppression of the expression of Bcl-2. Thus, manipulation of Bcl- 2 expression by targeting miR-143 could be used as a therapeutic strategy in cervical cancer treatment.

In conclusion, we demonstrated that miR-143 is downregulated in cervical cancer. Transfection experiments demonstrated that miR-143 serves as a tumor suppressor inhibiting HeLa cell proliferation and promoting apoptosis. miR-143 has the capacity to suppress the formation of tumor in nude mice. Mechanistically, these effects are Bcl-2-dependent. Therefore, miR-143 levels could be used as a biomarker in facilitating cervical tumor diagnosis. Finally, targeting miR-143 could be developed into a powerful therapeutic treatment for cervical cancer.

\section{Acknowledgements}

We thank Dr Zhang for providing the excellent technical assistance and helpful discussion. Members of the Department of Obstetrics and Gynecology, Xiangya Hospital, are acknowledged for their support and the material. This study was supported by grants from The National Natural Science Foundation of Hunan Province (no. 7JJ3058).

\section{References}

1. Meng F, Henson R, Wehbe-Janek H, et al: MicroRNA-21 regulates expression of the PTEN tumor suppressor gene in human hepatocellular cancer. Gastroenterology 133: 647-658, 2007.

2. Cordes KR, Sheehy NT, White MP, et al: miR-145 and miR-143 regulate smooth muscle cell fate and plasticity. Nature 460: 705-710, 2009.

3. Tang F, Kaneda M, O'Carroll D, et al: Maternal microRNAs are essential for mouse zygotic development. Genes Dev 21: 644-648, 2007.

4. Davidson MR, Larsen JE, Yang IA, et al: MicroRNA-218 is deleted and downregulated in lung squamous cell carcinoma. PLoS One 5: e12560, 2010

5. Giovannetti E, Funel N, Peters GJ, et al: MicroRNA-21 in pancreatic cancer: correlation with clinical outcome and pharmacologic aspects underlying its role in the modulation of gemcitabine activity. Cancer Res 70: 4528-4538, 2010. 
6. Hu G, Chen D, Li X, et al: miR-133b regulates the MET protooncogene and inhibits the growth of colorectal cancer cells in vitro and in vivo. Cancer Biol Ther 10: 190-197, 2010.

7. Motoyama K, Inoue $\mathrm{H}$, Mimori K, et al: Clinicopathological and prognostic significance of PDCD4 and microRNA-21 in human gastric cancer. Int J Oncol 36: 1089-1095, 2010.

8. Wickramasinghe NS, Manavalan TT, Dougherty SM, et al: Estradiol downregulates miR-21 expression and increases miR-21 target gene expression in MCF-7 breast cancer cells Nucleic Acids Res 37: 2584-2595, 2009.

9. Su H, Yang JR, Xu T, et al: MicroRNA-101, down-regulated in hepatocellular carcinoma, promotes apoptosis and suppresses tumorigenicity. Cancer Res 69: 1135-1142, 2009.

10. Liu X, Sempere LF, Galimberti F, et al: Uncovering growthsuppressive microRNAs in lung cancer. Clin Cancer Res 15: 1177-1183, 2009.

11. Sengupta S, den Boon JA, Chen IH, et al: MicroRNA 29c is downregulated in nasopharyngeal carcinomas, up-regulating mRNAs encoding extracellular matrix proteins. Proc Natl Acad Sci USA 105: 5874-5878, 2008

12. Wang X, Tang S, Le SY, et al: Aberrant expression of oncogenic and tumor-suppressive microRNAs in cervical cancer is required for cancer cell growth. PLoS One 3: e2557, 2008.

13. Martinez I, Gardiner AS, Board KF, et al: Human papillomavirus type 16 reduces the expression of microRNA-218 in cervical carcinoma cells. Oncogene 27: 2575-2582, 2008

14. Borralho PM, Kren BT, Castro RE, et al: MicroRNA-143 reduces viability and increases sensitivity to 5-fluorouracil in HCT116 human colorectal cancer cells. FEBS J 276: 6689-6700, 2009.

15. Kulda V, Pesta M, Topolcan O, et al: Relevance of miR-21 and miR-143 expression in tissue samples of colorectal carcinoma and its liver metastases. Cancer Genet Cytogenet 200: 154-160, 2010.

16. Gao W, Yu Y, Cao H, et al: Deregulated expression of miR-21, miR-143 and miR-181a in non-small cell lung cancer is related to clinicopathologic characteristics or patient prognosis. Biomed Pharmacother 64: 399-408, 2010.

17. Szczyrba J, Löprich E, Wach S, et al: The microRNA profile of prostate carcinoma obtained by deep sequencing. Mol Cancer Res 8: 529-538, 2010.

18. Zhang X, Liu S, Hu T, et al: Up-regulated microRNA-143 transcribed by nuclear factor kappa B enhances hepatocarcinoma metastasis by repressing fibronectin expression. Hepatology 50 : 490-499, 2009.
19. Clapé C, Fritz V, Henriquet C, et al: miR-143 interferes with ERK5 signaling, and abrogates prostate cancer progression in mice. PLoS One 4: e7542, 2009.

20. Ng EK, Tsang WP, Ng SS, et al: MicroRNA-143 targets DNA methyltransferases $3 \mathrm{~A}$ in colorectal cancer. Br J Cancer 101: 699-706, 2009.

21. Cheng AM, Byrom MW, Shelton J and Ford LP: Antisense inhibition of human miRNAs and indications for an involvement of miRNA in cell growth and apoptosis. Nucleic Acids Res 33: 1290-1297, 2005.

22. Yang Z, Chen S, Luan X, et al: MicroRNA-214 is aberrantly expressed in cervical cancers and inhibits the growth of HeLa cells. IUBMB Life 61: 1075-1082, 2009.

23. Pang RT, Leung CO, Ye TM, et al: MicroRNA-34a suppresses invasion through downregulation of Notch1 and Jagged1 in cervical carcinoma and choriocarcinoma cells. Carcinogenesis 31: 1037-1044, 2010.

24. Zhang H, Cai X, Wang Y, et al: microRNA-143, downregulated in osteosarcoma, promotes apoptosis and suppresses tumorigenicity by targeting Bcl-2. Oncol Rep 24: 1363-1369, 2010.

25. Pillai MR, Halabi S, McKalip A, et al: The presence of human papillomavirus-16/-18 E6, p53, and Bcl-2 protein in cervicovaginal smears from patients with invasive cervical cancer. Cancer Epidemiol Biomarkers Prev 5: 329-335, 1996

26. Dimitrakakis C, Kymionis G, Diakomanolis E, et al: The possible role of p53 and bcl-2 expression in cervical carcinomas and their premalignant lesions. Gynecol Oncol 77: 129-136, 2000.

27. Xiong Y, Fang JH, Yun JP, et al: Effects of microRNA-29 on apoptosis, tumorigenicity, and prognosis of hepatocellular carcinoma. Hepatology 51: 836-845, 2010.

28. Chen G, Zhu W, Shi D, et al: microRNA-181a sensitizes human malignant glioma U87MG cells to radiation by targeting Bcl-2. Oncol Rep 23: 997-1003, 2010.

29. Xia L, Zhang D, Du R, et al: miR-15b and miR-16 modulate multidrug resistance by targeting BCL2 in human gastric cancer cells. Int J Cancer 123: 372-379, 2008. 